\title{
Gravity Attraction as a Result of Resonant Interaction between Standing Quantum Recurrences
}

\section{A.A.Berezin}

Independent researcher, Moscow, Russia

\section{*Correspondence author}

\author{
A.A.Berezin \\ Independent researcher \\ Moscow \\ Russia
}

Submitted : 25 Jan 2021 ; Published : 13 Feb 2021

\begin{abstract}
It has been shown theoretically that a gravity force can be formed between two phonon generators through resonant interacting between their phonon wave functions-standing recurrences in the medium of the physical vacuum. Following the theoretical model, an experimental gravity generator was developed based on a massive ferromagnetic toroid core with a coil.
\end{abstract}

Keywords: Gravitational Force, Phonon Generators, Fermi-Pasta-Ulam Recurrence, Phonon Wave Functions-standing Recurrence in the Medium of the Physical Vacuum.

Introduction

The works of Isaac Newton and Albert Einstein dominate the development of gravitational theory. Newton's classical theory of gravitational force held sway from his Principia, published in 1687, until Einstein's work in the early 20th century. Even today, Newton's theory is of sufficient accuracy for all but the most precise applications. Einstein's modern field theory of general relativity predicts only minute quantitative differences from the Newtonian theory except in a few special cases. The major significance of Einstein's theory is its radical conceptual departure from classical theory and its implications for further growth in physical thought.

The Newtonian theory of gravity is based on an assumed force acting between all pairs of bodies - i.e., an action at a distance. When a mass moves, the force acting on other masses has been considered to adjust instantaneously to the new location of the displaced mass. Special relativity theory states that no physical signal travels faster than the speed of light and that all signals travel at this speed through empty space. This theory, with the field theory of electrical and magnetic phenomena, has met such empirical success that most modern gravitational theories are constructed as field theories consistent with the principles of special relativity. In a field theory the gravitational force between bodies is formed by a two-step process:

1. One body produces a gravitational field that permeates all surrounding space but has weaker strength farther from its source. A second body in that space is then acted upon by this field and experiences a force.

2. The Newtonian force of reaction is then viewed as the response of the first body to the gravitational field produced by the second body, there being at all points in space a superposition of gravitational fields due to all the bodies in it [1].

According to the Newtonian theory of gravity the gravitational force between two bodies is:

$$
F_{12}=\frac{G M_{1} M_{2}}{r_{12}^{2}}
$$

Where $M_{1}, M_{2}$ are two masses separated by distance $r_{12}$ the constant $\mathrm{G}$ is a quantity with the physical dimensions $(\text { lenght })^{3} /($ mass $)(\text { time })^{2}$.

\section{Model of the phonon standing quantum recurrence in a crystalline lattice}

The purpose of the paper is to assess a possibility of changing the magnitudes of masses by adding or subtracting the sums of effective phonon gas masses $\sum m_{\text {leffect }}, \sum m_{\text {2effect }}$, created in the crystalline lattices of bodies thus changing their attracting force. As a result the gravitation force equation (1) will look like follows:

$$
F_{12}=\frac{G\left(M_{1} \pm \sum m_{1 \text { effect }}\right)\left(M_{2} \pm \sum m_{2 \text { effect }}\right)}{r_{12}^{2}}
$$

As it was shown [2] the energy spectrum of a quantum particle in a periodical field has a zone structure. Consider a one dimensional crystal with a lattice period equal to a. $\psi(x)$ Let be a solution to the Shrodiger equation with a periodical potential energy and $\mathrm{E}=\hbar \omega$ is the energy of a phonon in the state $\psi(x)$ :

$$
-\frac{\hbar^{2}}{2 m} \frac{\partial^{2} \psi(x)}{\partial x^{2}}=E \psi(x)
$$


The wave function of a phonon looks like:

$$
\psi(x)=\psi_{k}(x)=\varphi_{k}(x) e^{i k x}
$$

Therefore, the wave function $\psi_{k+2 \pi / a}(x)$ is:

$$
\psi_{k+2 \pi / a}(x)=\varphi_{k+2 \pi / a}(x) e^{2 \pi k i / a} e^{i k x}
$$

As it goes from (4) and (5) independently of the shape of the periodical field the solutions of (3) form a number of arrays. In any of them, it is possible to consider that the wave vector variates within a following segment $-\pi / a \leq k \leq \pi / a$. One of the arrays is composed by all wave functions $\psi_{k+2 m / a}(x)$ under a fixed meaning of the number $n$.

The energy spectrum of a particle (in our case of a phonon) in a periodical field has a zone stucture. One can see that near the borders of the zones $\frac{\partial E}{\partial k}=0$ because when $k=0$ and

$k= \pm \pi / a$ the wave function of a phonon is a standing wave. Since a group velocity of a standing wave is zero the following equality is rightful:

$$
\frac{\partial E}{\partial k}=\hbar \frac{\partial \omega}{\partial k}=\hbar V_{\text {group }}=0
$$

Expanding $\mathrm{E}(\mathrm{k})$ within the region $\mathrm{k}=0$ into the Tailor sequence and taking its first two members we can get:

$$
E(k)-E(0)=\frac{\hbar^{2} k^{2}}{2 m^{*}}
$$

Where $m^{*}-$ is the effective mass and

$$
\frac{1}{m^{*}}=\frac{1}{\hbar} \frac{\partial^{2} E(0)}{\partial k^{2}}
$$

As it can be seen from (8) the effective mass is inversely proportional to the curvature of the curve $\mathrm{E}(\mathrm{k})$ at the border of the zone. That is why the absolute magnitude of the effective mass is large in narrow zones and small in wide ones. The effective mass is positive at the bottom of the allowed zone and negative near its upper cover.

The concept of icreasing mass through enhancing the energy of phonons in a crystalline lattice of a solid body by placing a vibrator into it was first considered by a British scientist A.B.Pippard [3].

To assess the possibility of changing masses of the crystalline lattice atoms we'll use the dispersion ratio for spatial waves in there which looks like:

$$
\omega=2 \sqrt{\frac{\chi}{m_{\text {atom }}}}\left|\sin \frac{k a}{2}\right|
$$

where $\chi$ is the elasticity coefficient of the force linking neighbouring atoms in a crystalline lattice, $m_{\text {atom }}$ is the mass of the atom.

The energy of phonons accounting (9) can be written as:

$$
E_{p h}=2 \hbar \sqrt{\frac{\chi}{m_{a t o m}}}\left|\sin \frac{p a}{2 \hbar}\right|
$$

Where $\mathrm{p}-$ is the impulse

It is evident that one needs to change the dispersion ratio (9) to increase the energy of phonons in a crystalline lattice. A possible way to do it is to form a selfoscillating quantum process there. Using the approach proposed by A.B.Pippard [3] and an analogy with a delayed selfoscillating system [4] a new dispersion ratio can be written like follows:

$$
\omega=2 \sqrt{\frac{\chi}{m_{\text {atom }}}}\left|\operatorname{ctg} \frac{k a}{2}\right|
$$

A.B.Pippard [3] also showed that a resonant increasing of the density of states in a crystalline lattice brings a mass increase of a solid body [5].

To form a new dispersion ratio (11) it is necessary to provide a spatial delay for a quantum oscillator. For that purpose a ferromagnetic with a very high initial permeability can be used. If to make a quasi static magnetization process in the ferromagnetic by traveling only along the partial hysteresis loops we can get a physical analog of the delayed Shrodinger equation solution. Consider this form of the Shrodinger equation which corresponds to the dispersion ratio (11):

$$
\begin{aligned}
& -\frac{\hbar^{2}}{2 m} \frac{d^{2} \psi(x)}{d x^{2}}+d_{0} \frac{d \psi(x)}{d x}(1-Z)=E \psi(x) \\
& \left.L_{n}\left|\operatorname{ctg} \frac{x a+\xi_{1}}{2}\right|+1\right) \frac{d Z}{d x}+Z=|\psi(x)|^{2}
\end{aligned}
$$

where $\mathrm{Z}$ is proportional to the phonon density in the $n^{\text {th }}$ sliced region of the ferromagnetic toroid, covered by the $n^{\text {th }}$ turn of the toroidal coil, placed on the ferromagnetic, $\mathrm{L} n-$ is proportional to the square of the $n^{\text {th }}$ local hysteresis loop $\int H d B$ in the $n^{\text {th }}$ sliced region of the ferromagnetic, induced by a low frequency quasi constant small current in the toroidal coil, $d$ $<1, \frac{\partial \psi(x)}{\partial x}$ describes a dispersion process taking place due to the alternation of turns and empty spaces on the ferromagnetic core, $\xi_{1}$ is a random function distributed within the interval $[\pi / 2 ; \pi / 2]$ reflecting a probabilistic traveling process along the local (not the main one) hysteresis loops.

The solution of the system (12) represents a sum of different energy states but differs from a classical case because the frequencies are not equidistant and form a so called dispersion sequence:

$$
E_{1}=\sum_{n=1}^{\infty} \sin \omega_{n} \hbar
$$

where the frequencies $\omega_{\mathrm{n}}$ are formed by the dispersion ratio (11) $E_{1}$ is the sum energy of the phonon non ideal gas in the delayed lattice.

So a spacially delayed Shrodinger equation (12) by analogy with the Van der Pol's equation describes a phonon gas generating process in the toroidal ferromagnetic structure. It is necessary to emphasize that together with (13) an equidistant sequence of frequencies and corresponding levels of energy of the phonon dynamics exist in the crystalline lattice of the ferromagnetic core satisfing the Shrodinger equation (3):

$$
E_{2}=\sum_{m=1}^{\infty} n \omega \hbar
$$

As it was shown [6] the presence of two types of energy levels (13), (14) in a periodical structure forms a recurrence of the energy regrouping between (13) and (14). Hence unifying (3) and (12) we come to a system that describes the dynamics of the phonon gas in the structure of the ferromagnetic core: 
$-\frac{\hbar^{2}}{2 m} \frac{d^{2} \psi_{1}(x)}{d x^{2}}=\psi_{1}(x)\left(1+\psi_{2}(x)\right)$

$-\frac{\hbar^{2}}{2 m} \frac{d^{2} \psi_{2}(x)}{d x^{2}}+d_{0} \frac{d \psi_{2}(x)}{d x}(1-Z)=\psi_{2}(x)\left(1+\psi_{1}(x)\right)$

$L_{n}\left(\left|\operatorname{ctg} \frac{x a+\xi_{1}}{2}\right|+1\right) \frac{d Z}{d x}+Z=\left|\psi_{2}(x)\right|^{2}$

where $\xi_{1}$ is a radom function distributed within the interval $[-\pi / 2, \pi / 2]$

A numerical study of the system (15) shows that its solution demonstrates a probabilistic recurrence to its initial state but differently from the classical Fermi-Pasta-Ulam recurrence [8], in the system (15) we are dealing with a quantum or probabilistic recurrence [7]. As it can be seen in Fig.1-4. The Fourier spectrum of the wave function $\psi_{1}(x)$ recurs to its initial state. Different from the classical FPU recurrence [8] the quantum one requires developing a number of intermediate recurrences to reach a relatively stable similarity between chosen Fourier images in the sequence. This process may have different scenarios but their number according to the numerical study is limited to $10-12$.

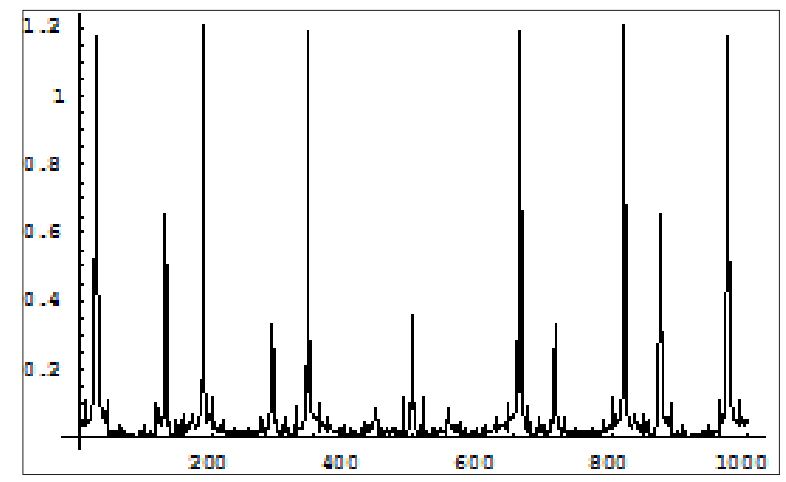

Figure 1: Initial Fourier image of the wave function $\psi_{1}(x)$ in the solution of (15). Ver. axis - amplitude, horiz. axis - spatial coordinate (units are conditional)

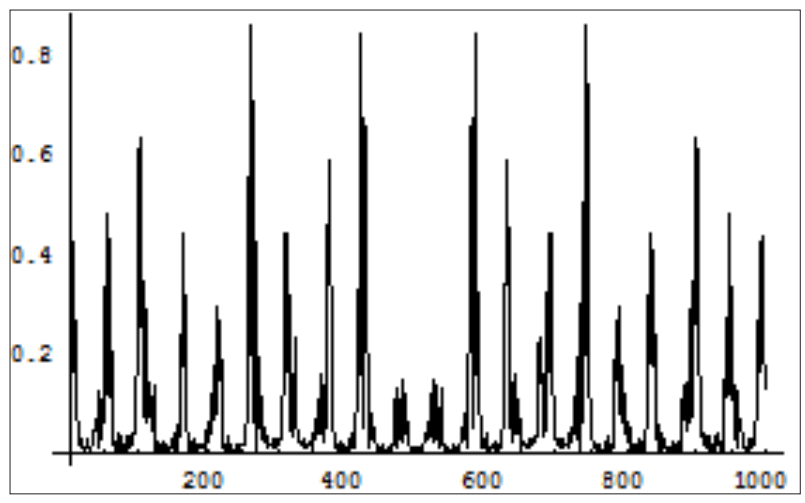

Figure 2: First intermediate Fourier image of the wave function $\psi_{1}(x)$ in the solution of (15). Ver. axis - amplitude, horiz. axis - spatial coordinate (units are conditional)

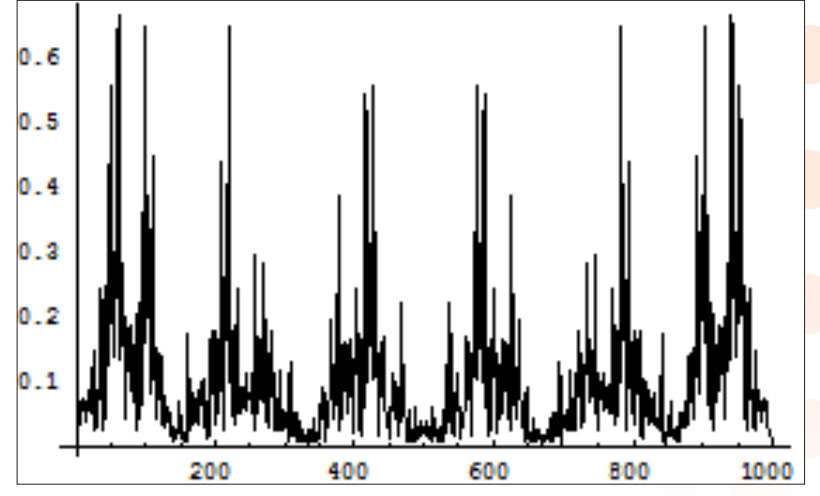

Figure 2: Second intermediate Fourier image of the wave function $\psi_{1}(x)$ in the solution of (15). Ver. axis - amplitude,

horiz. axis - spatial coordinate (units are conditional)

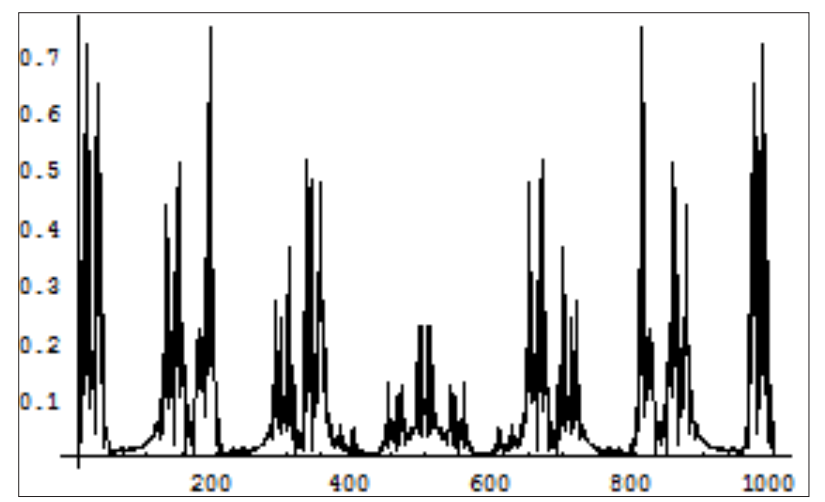

Figure 3: Third intermediate Fourier image of the wave function $\psi_{1}(x)$ in the solution of (15). Ver. axis - amplitude, horiz. axis - spatial coordinate (units are conditional)

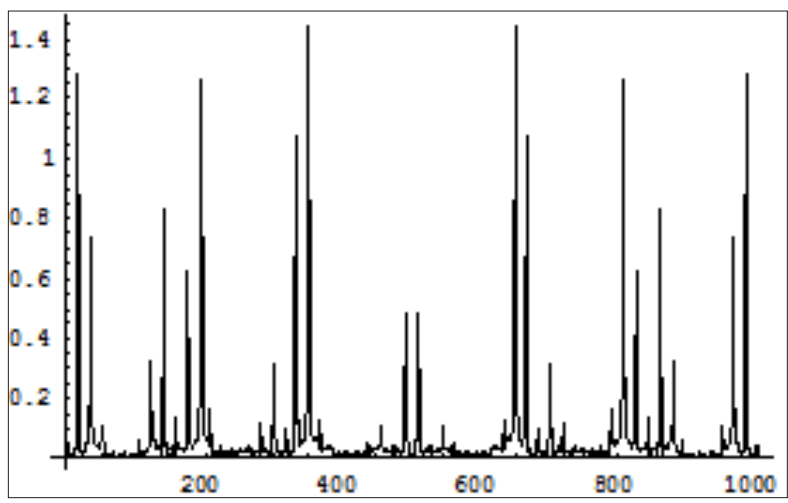

Figure 4: Fourth intermediate Fourier image of the wave function $\psi_{1}(x)$ in the solution of (15). Ver. axis - amplitude, horiz.

Axis - spatial coordinate (units are conditional)

As it can be seen from Fig. 1 - 4 the type of recurrence under study refers to a spatial one and develops among interacting standing waves. A physical model of the standing recurrence can be obtained in a toroidal ferromagnetic core randomly magnetized along only local hysteresis loops.

Triangular model of the phonon standing quantum recurrence in a crystalline lattice of the phonon generator ferromagnetic core

For a toroidal form of the phonon generator we have to con- 
sider at least three coupled systems like (15) forming a closed ring in which the standing wave functions can be formed. The triangular system looks as follows:

$-\frac{\hbar^{2}}{2 m} \frac{d^{2} \psi_{1}(x)}{d x^{2}}=\psi_{1}(x)\left(1+\psi_{2}(x)+\psi_{6}(x)\right)$

$-\frac{\hbar^{2}}{2 m} \frac{d^{2} \psi_{2}(x)}{d x^{2}}+d_{0} \frac{d \psi_{2}(x)}{d x}(1-Z)=\psi_{2}(x)\left(1+\psi_{1}(x)+\psi_{3}(x)\right)$

$L_{n}\left(\left|\operatorname{ctg} \frac{x a+\xi_{1}}{2}\right|+1\right) \frac{d Z}{d x}+Z=\left|\psi_{2}(x)\right|^{2}$

$-\frac{\hbar^{2}}{2 m} \frac{d^{2} \psi_{3}(x)}{d x^{2}}=\psi_{3}(x)\left(1+\psi_{4}(x)+\psi_{2}(x)\right)$

$-\frac{\hbar^{2}}{2 m} \frac{d^{2} \psi_{4}(x)}{d x^{2}}+d_{0} \frac{d \psi_{4}(x)}{d x}(1-P)=\psi_{4}(x)\left(1+\psi_{3}(x)+\psi_{5}(x)\right)$

$L_{n}\left(\left|\operatorname{ctg} \frac{x a+\xi_{1}}{2}\right|+1\right) \frac{d P}{d x}+P=\left|\psi_{4}(x)\right|^{2}$

$-\frac{\hbar^{2}}{2 m} \frac{d^{2} \psi_{5}(x)}{d x^{2}}=\psi_{5}(x)\left(1+\psi_{6}(x)+\psi_{4}(x)\right)$

$-\frac{\hbar^{2}}{2 m} \frac{d^{2} \psi_{6}(x)}{d x^{2}}+d_{0} \frac{d \psi_{6}(x)}{d x}(1-Y)=\psi_{6}(x)\left(1+\psi_{5}(x)+\psi_{1}(x)\right)$

$L_{n}\left(\left|\operatorname{ctg} \frac{x a+\xi_{1}}{2}\right|+1\right) \frac{d Y}{d x}+Y=\left|\psi_{6}(x)\right|^{2}$

The system (16) contains two additional couples of wave functions: $\psi_{3}, \psi_{4}, \psi_{5}, \psi_{6}$.

The constant coefficients in the system (16) are omitted for simplicity.

$\mathrm{F}_{1}, \mathrm{~F}_{2}$ are the high and low frequency Gaussian random functions reflecting micro and macro thermal vibrations in the lattice.

The system (16) represents a simplified model of a phonon generator, consisting of three coupled couples of the high frequency wave, reflecting the dynamics of phonons in the crystalline lattice of the toroidal copper wire coil interacting with three coupled couples of the delayed wave functions created in the crystalline lattice of the toroidal ferromagnetic core.

A numerical study of the system (16) shows that its solution displays a new form of quantum recurrence - a standing one. Differ from the classical Fermi-Pasta-Ulam recurrence the obtained one in the system (16) demonstrates only a phase and amplitude recurrence to the initial states. As it can be seen in Fig.5-7. Only phases and amplitudes of the Fourier spectra of the wave functions $\psi_{1}(x), \psi_{2}(x), \psi_{3}(x)$ recur to their initial states. In case of the standing quantum recurrence the Fourier spectrum of the wave function remains quasi stable whereas one can observe a recurrence of the amplitude magnitude and a quasi periodical phase variation of the probability spectrum harmonics $\left|\psi_{1}(x)\right|^{2}$. In fact a standing recurrence arises as a result of a nonlinear interaction between the high frequency phonon oscillations having the dispersion ratio (9) and the low frequency phonon oscillations with the dispersion ratio (11). In a general case accounting (9) and (11) one can conclude that the standing recurrence has no certain spatial scale which is imposed on the system only by the dimensions of the phonon generators under consideration (even for example stars and planets) because the uncertainty principle in a case of the cotangent function (11) differs from that of the sinus one, typical for the diffraction picture which is: $\sin ^{2} k a / k a^{2}$. For a cotangent function it looks like in Fig.5.

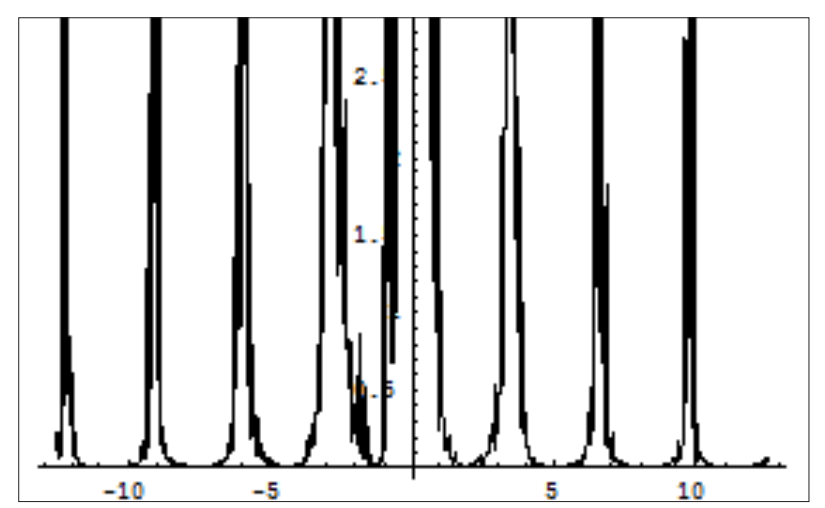

Figure 5: Cotangent function $c^{2} g^{2} k a / k^{2}$ having a randomly changing phase. Horiz. Axis- distance divisible by a.

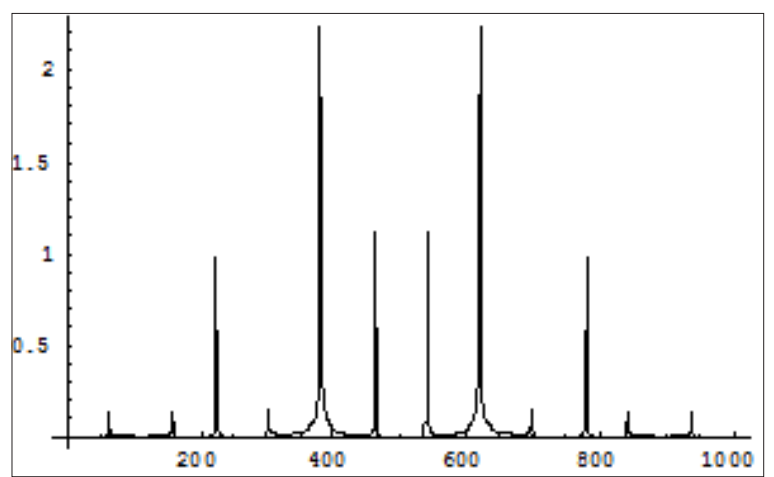

Figure 6

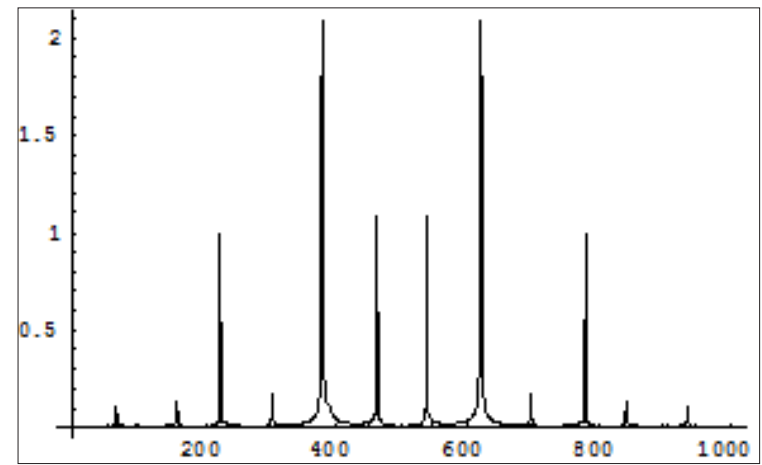

Figure 7

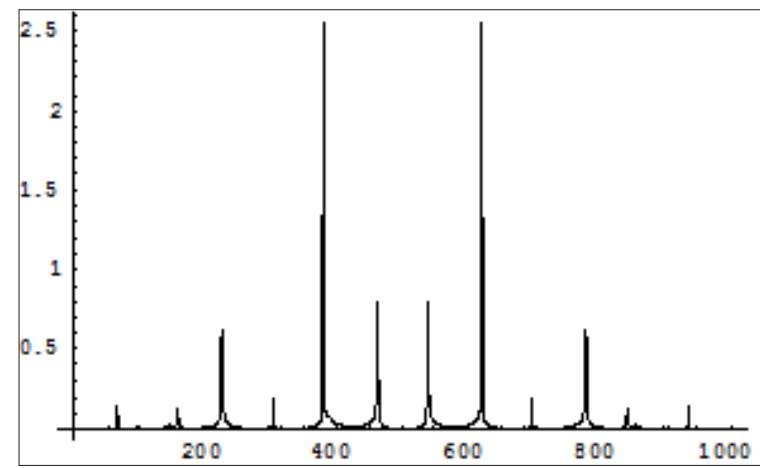

Figure 8 


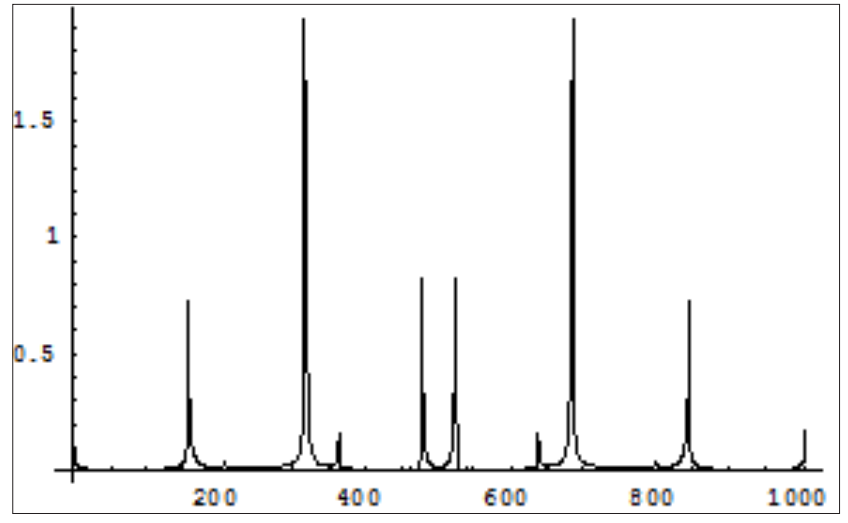

Figure 9



Figure 10



Figure 11

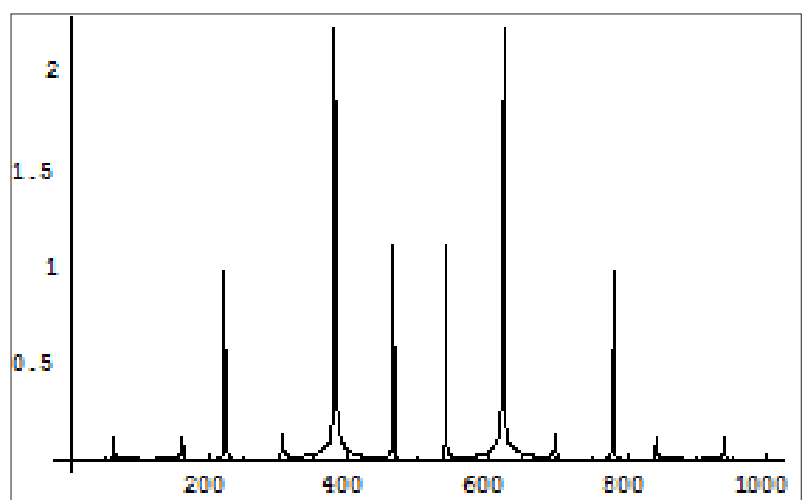

Figure 12

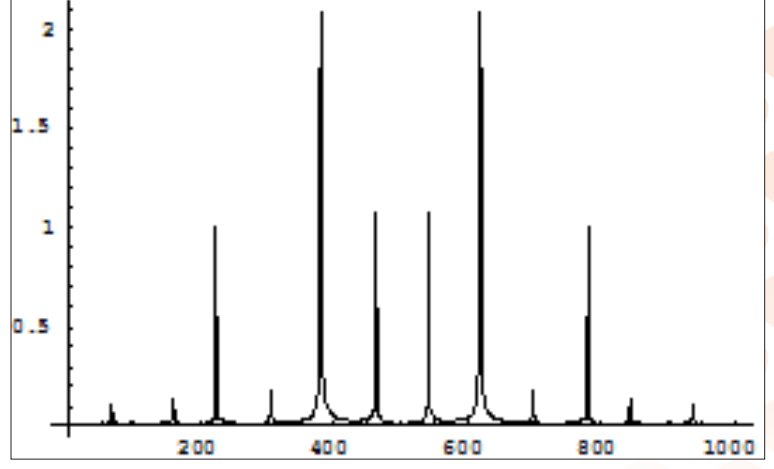

Figure 13

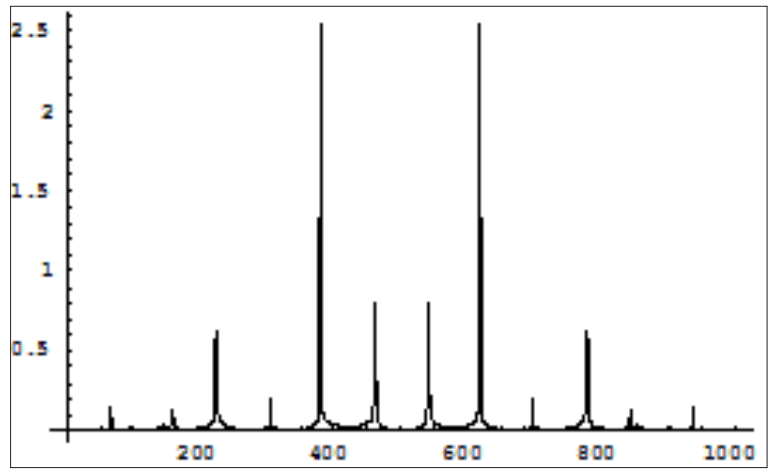

Figure 14

Figures $6,7,8,9,10,11,12,13,14$ display in sucsession the standing spatial recurrence in the coupled triangular system (16). As it can be seen from the graphs, the standing recurrence represents only a quasi periodical variation of amplitudes and phases around their average magnitudes.

Consider now a process of interaction between quantum standing recurrences of the phonon wave functions generated inside a toroidal ferromagnetic core with a coil on it and another phonon generator described within the framework of the system (16). Assume that a medium for possible existance of such recurrences is a physical vacuum or a space where all particles are spread up. According to the standing character of the recurrences, their spatial waves don't propagate. Their interaction represents a mutual phase tuning or «tutouring each other» after which a mutual resonance [9] is formed regardless the distance between them. The installed resonance provides an instantaneous interaction between the two phonon generators.

First we suppose that together with a traditional virtual reaction between an electron-positron couple and two photons in the physical vacuum there exists an analogous electron-positron annihilation act giving a birth to two phonons which looks as follows:

$$
e^{-}+e^{+} \leftrightarrow \text { phonon }+ \text { phonon }
$$

We consider the physical vacuum as an infinite-dimensional system consisting of interacting virtual reactions (17). The distance between the toroidal phonon standing recurrence generator and another phonon generator will be R. So the phonon standing recurrence field interaction dynamics will be developing in a sphere having radius $\mathrm{R}$. To describe this process we'll 
use the approach suggested by Saltzman and Lorenz [10,11]. We'll model the motion of the phonon wave functions phases in the physical vacuum within the framework of the following equation:

$$
\frac{\partial Q\left(X, V_{x}\right)}{\partial V_{x}}=L(X) Q\left(X, V_{x}\right)
$$

Where $\mathrm{Q}$ is an $\mathrm{M}$ - dimentional vector that specifies the state of the physical vacuum quantum fluid, consisting of all possible particles energy states $V_{x}$, - is the phonon wave function spatial phase velocity, $\mathrm{X}$ - is the configuration space (with components $\mathrm{x}, \mathrm{y}, \mathrm{Z}$ in three dimensions and $\mathrm{L}(\mathrm{X})$ - is a time-independant, nonlinear differential operator.

A common technique in studying the behavior of (18) is to Fourier analyze Q into mode amplitudes:

$$
Q\left(X, V_{x}\right)=\sum_{k} q_{k}\left(V_{x}\right) e^{i k x}
$$

where

$q_{k}\left(V_{x}\right)=\frac{1}{(2 \pi)^{3}} \int d^{3} x Q\left(X, V_{x}\right) e^{-i k x}$

Inserting ((19) in (18) and using the orthogonality of the exponental functions $e^{i k x}$ we obtain for each mode $\mathrm{q}_{k}$ an equation in the form:

$\frac{d q_{k}}{d V_{x}}=V_{k}\left(q_{1} \ldots q_{k}\right)$

If only the $\mathrm{N}$ «most important modes» in the sum (19) are kept then (21) represents a set of MN first-order ordinary differential eqations describing the evolution of the mode amplitude components against the phonon wave function spatial phase velocity $V_{x}$. This procedure of Fourier analysis followed by truncation is often called the Garerkin approximation.

Suppose that the interaction process between the energies of phonons in the generator and that of the physical vacuum will take a character similar to the process of thermoconvection. That gives a possibility to use the model of the Rayleigh-Benard convection. Worth emphasizing that in the case under study, we are dealing with two types of uncertainty: first refered to the dimension of the crystalline lattice of the generator material and the second one refered to the considered volume of the space (in our case this is a sphere having a radius $\mathrm{R}$ ).

Consider the interaction dynamics similar to that of convection in a spherical layer having a width $\mathrm{R}$ (distance in the vacuum between two phonon generators). Interaction takes place in the shaded circular reagions in the Fig. 15 as it follows from the dispersion ratio (11) with a random phase (Fig.5.).

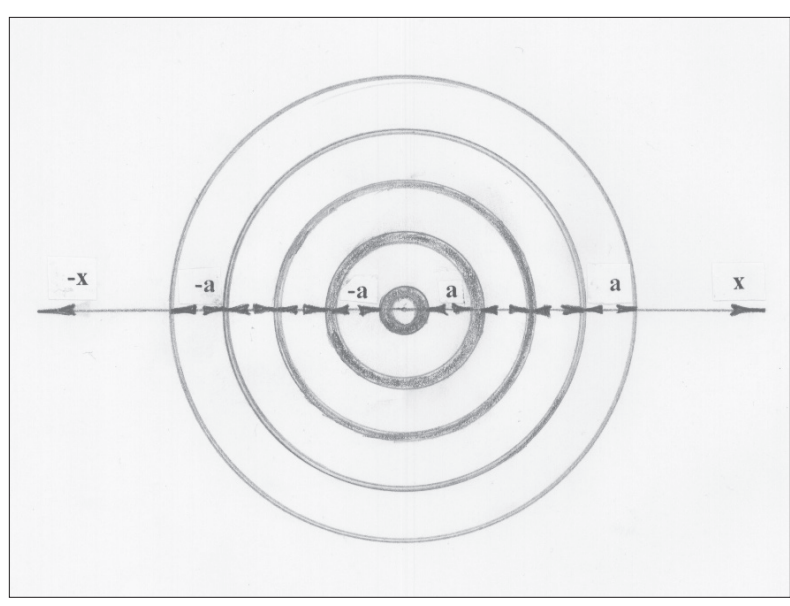

Figure 15: Interaction reagions of phonon generators corresponding to the dispersion ratio (11).For a microscale a - is the crystalline lattice constant of the phonon generator material. For a macroscale (1) the length a is equal to the diameter of the planet.

Skipping the procedure of reducing (it can be found in [12]) we get the Lorenz system of equations:

$$
\begin{aligned}
& \frac{d u}{d x}=-\sigma x+\sigma y \\
& \frac{d v}{d x}=-u w+\beta u-v \\
& \frac{d w}{d x}=u v-b w
\end{aligned}
$$

Where

$\mathrm{u}$ - is the energy of the attractor formed in the vacuum as a result of interaction between phonons of the generator and those of the vacuum,

$\mathrm{v}$ - is the difference between the energies at the borders of the spherical layer of interaction (Fig.15),

$\mathrm{w}-$ is the distribution of the energy states along the spherical layer.

$\mathrm{x}$ - is the spacial coordinate normalized to the phase velocity $V_{x}, \sigma$ - is the Pradtl number analog which is close to 1 ,

$\beta$ - is the dimensionless measure of the energies difference at the borders of the spherical layer of interaction (normalized analog of the Rayleigh number),

b- is the geometrical factor divisible by $\mathbf{a}$.

To form a full model of interaction between the two phonon standing recurrence generators through the medium of the physical vacuum the two systems (16) and (22) are coupled and added by another system similar to (16) but having no external sinusoidal perturbation function because any solid body objects can be considered as phonon generators. The obtained system looks in the following way:

$$
\begin{aligned}
& -\frac{\hbar^{2}}{2 m} \frac{d^{2} \psi_{1}(x)}{d x^{2}}=\psi_{1}(x)\left(1+\psi_{2}(x)+\psi_{6}(x)\right)+\sin k_{0} x+F_{1}+F_{2} \\
& -\frac{\hbar^{2}}{2 m} \frac{d^{2} \psi_{2}(x)}{d x^{2}}+d_{0} \frac{d \psi_{2}(x)}{d x}(1-Z)=\psi_{2}(x)\left(1+\psi_{1}(x)+\psi_{3}(x)\right)+\sin k_{0} x+F_{1}+F_{2} \\
& \left.L_{n}\left|\operatorname{ctg} \frac{x a+\xi_{1}}{2}\right|+1\right) \frac{d Z}{d x}+Z=\left|\psi_{2}(x)\right|^{2}
\end{aligned}
$$




$$
\begin{aligned}
& -\frac{\hbar^{2}}{2 m} \frac{d^{2} \psi_{3}(x)}{d x^{2}}=\psi_{3}(x)\left(1+\psi_{4}(x)+\psi_{2}(x)\right)+\sin k_{0} x+F_{1}+F_{2} \\
& -\frac{\hbar^{2}}{2 m} \frac{d^{2} \psi_{4}(x)}{d x^{2}}+d_{0} \frac{d \psi_{4}(x)}{d x}(1-P)=\psi_{4}(x)\left(1+\psi_{3}(x)+\psi_{5}(x)\right)+\sin k_{0} x+F_{1}+F_{2} \\
& L_{n}\left(\left|\operatorname{ctg} \frac{x a+\xi_{1}}{2}\right|+1\right) \frac{d P}{d x}+P=\left|\psi_{4}(x)\right|^{2} \\
& -\frac{\hbar^{2}}{2 m} \frac{d^{2} \psi_{5}(x)}{d x^{2}}=\psi_{5}(x)\left(1+\psi_{6}(x)+\psi_{4}(x)\right)+\sin k_{0} x+F_{1}+F_{2} \\
& -\frac{\hbar^{2}}{2 m} \frac{d^{2} \psi_{6}(x)}{d x^{2}}+d_{0} \frac{d \psi_{6}(x)}{d x}(1-Y)=\psi_{6}(x)\left(1+\psi_{5}(x)+\psi_{1}(x)\right)+\sin k_{0} x+F_{1}+F_{2} \\
& \left.L_{n}\left|\operatorname{ctg} \frac{x a+\xi_{1}}{2}\right|+1\right) \frac{d Y}{d x}+Y=\left|\psi_{6}(x)\right|^{2} \\
& \frac{d u(x)}{d x}=(-\sigma u(x)+\sigma(x))\left(1+\psi_{5}(x)+\psi_{4}(x)\right) \\
& \frac{d v(x)}{d x}=-u(x) w(x)+\beta u(x)\left(1+\psi_{1}(x)+\psi_{6}(x)-v(x)\right. \\
& \frac{d w(x)}{d x}=u(x) v(x)-b w(x)\left(1+\psi_{1}(x)+\psi_{3}(x)\right) \\
& -\frac{\hbar^{2}}{2 m} \frac{d^{2} \psi_{7}(x)}{d x^{2}}=\psi_{7}(x)\left(1+\psi_{8}(x)+\psi_{12}(x)\right)+w(x)+F_{3}+F_{4} \\
& -\frac{\hbar^{2}}{2 m} \frac{d^{2} \psi_{8}(x)}{d x^{2}}+d_{0} \frac{d \psi_{8}(x)}{d x}(1-Z)=\psi_{8}(x)\left(1+\psi_{7}(x)+\psi_{9}(x)\right)+F_{3}+F_{4} \\
& L_{n}\left(\xi_{2}+1\right) \frac{d Z}{d x}+Z=\left|\psi_{8}(x)\right|^{2} \\
& -\frac{\hbar^{2}}{2 m} \frac{d^{2} \psi_{9}(x)}{d x^{2}}=\psi_{9}(x)\left(1+\psi_{10}(x)+\psi_{8}(x)\right)+u(x)+F_{3}+F_{4} \\
& -\frac{\hbar^{2}}{2 m} \frac{d^{2} \psi_{10}(x)}{d x^{2}}+d_{0} \frac{d \psi_{10}(x)}{d x}(1-P)=\psi_{10}(x)\left(1+\psi_{9}(x)+\psi_{11}(x)\right)+F_{3}+F_{4} \\
& L_{n}\left(\xi_{2}+1\right) \frac{d P}{d x}+P=\left|\psi_{10}(x)\right|^{2} \\
& -\frac{\hbar^{2}}{2 m} \frac{d^{2} \psi_{11}(x)}{d x^{2}}=\psi_{11}(x)\left(1+\psi_{12}(x)+\psi_{10}(x)\right)+\chi(x)+F_{3}+F_{4} \\
& -\frac{\hbar^{2}}{2 m} \frac{d^{2} \psi_{12}(x)}{d x^{2}}+d_{0} \frac{d \psi_{12}(x)}{d x}(1-Y)=\psi_{12}(x)\left(1+\psi_{11}(x)+\psi_{7}(x)\right)+F_{3}+F_{4} \\
& L_{n}\left(\xi_{2}+1\right) \frac{d Y}{d x}+Y=\left|\psi_{12}(x)\right|^{2}
\end{aligned}
$$

Where $\psi_{7}, \psi_{8}, \psi_{9}, \psi_{10}, \psi_{11}, \psi_{12}$ are the wave functions of the second phonon generator, $\xi_{2}$ is a random function uniformly distributed within the interval $[-0.5 ; 0.5]$ reflecting the non regularities in the crytalline lattice of the second phonon generator, are the high and low frequency Gaussian random functions reflecting micro and macro thermal vibrations in the lattice of the second phonon generator.

System (23) describes a process of interaction between the quantum standing recurrences of the phonon wave functions generated inside a toroidal ferromagnetic core with a coil on it (as well as in any other phonon generator including a planet) and another phonon generator (a piece of metal or a planet) through a medium of the physical vacuum.

Computer study of the system (23) shows that the standing recurrences of two phonon generators interact despite the random character of the medium $(17,22)$. The solutions of (23) display a limited number of scenarios in which one can see that a generator possessing a greater number of energy states «leads» the generator with a lower quantity of states. The «governed» generator has a «blurred» form of a standing recurrence. The following graphs demonstrate the most typical snapshots of the interaction process.

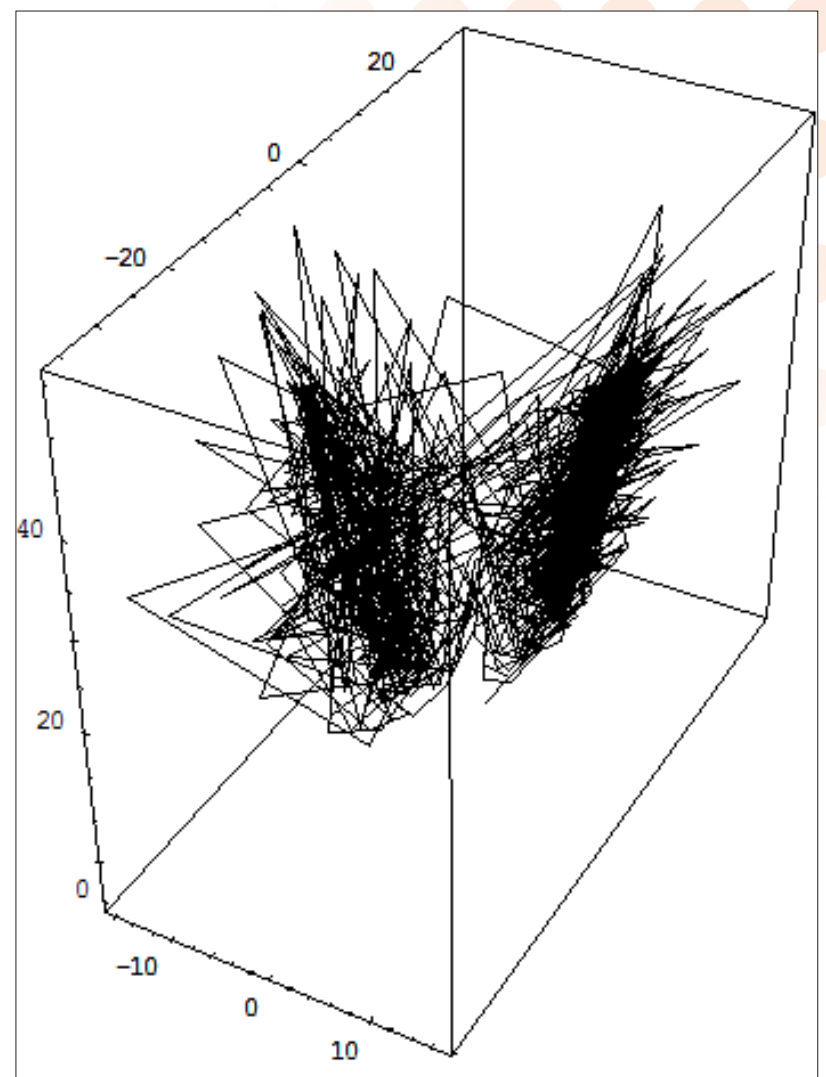

Figure 16: The $3 \mathrm{D}$ image of the functions $u, v, w$ in the system (23).

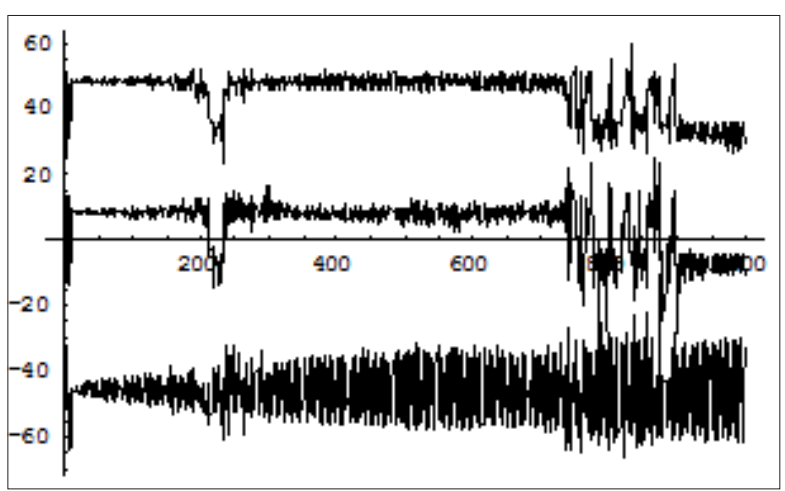

Figure 17: The functions $u, v, w$ in the system (23).

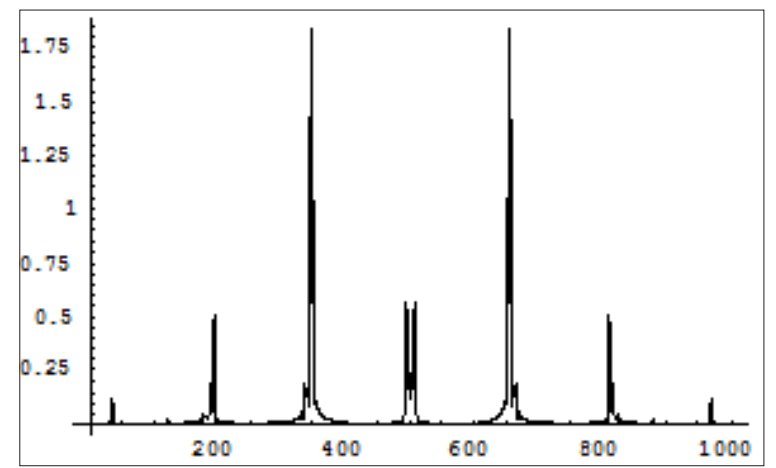

Figure 18 


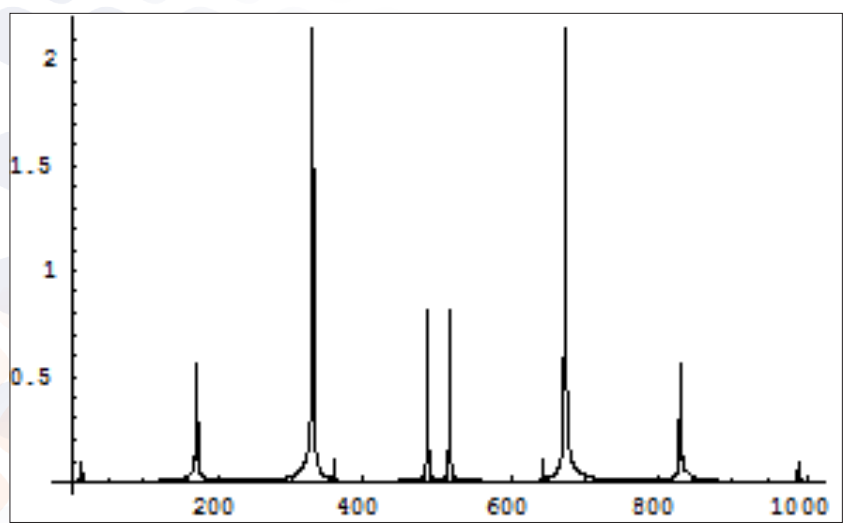

Figure 19

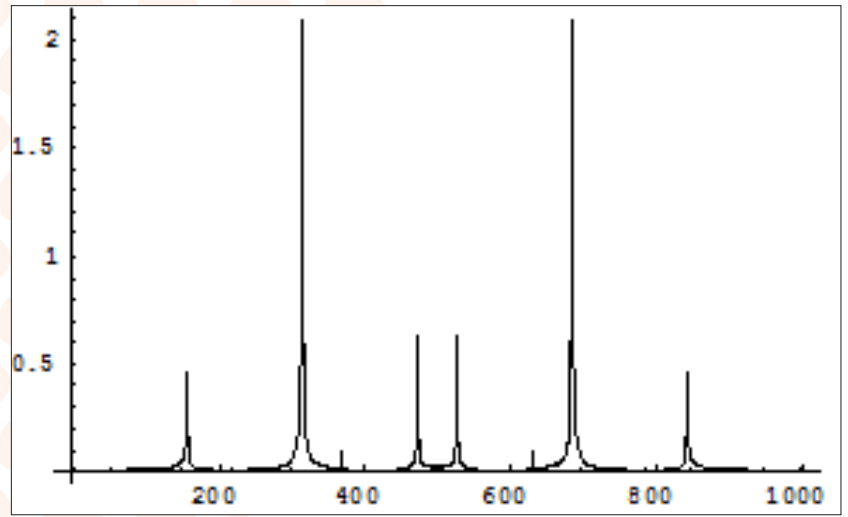

Figure 20

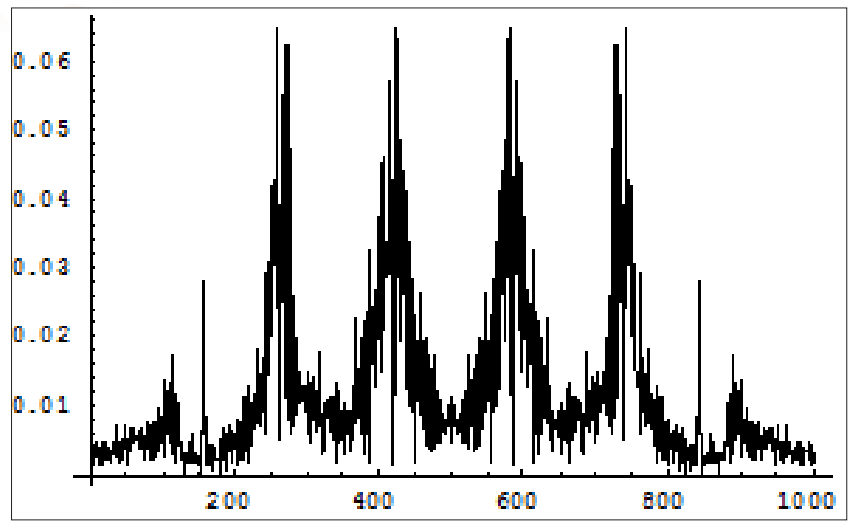

Figure 21

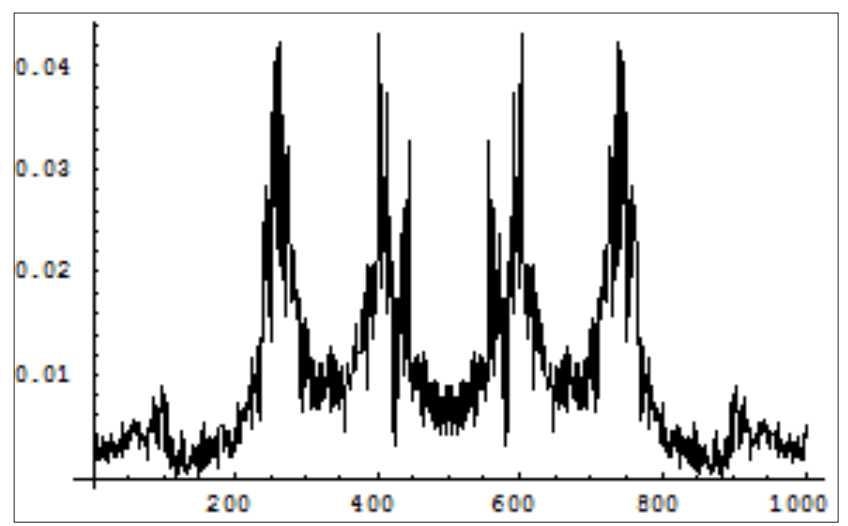

Figure 22

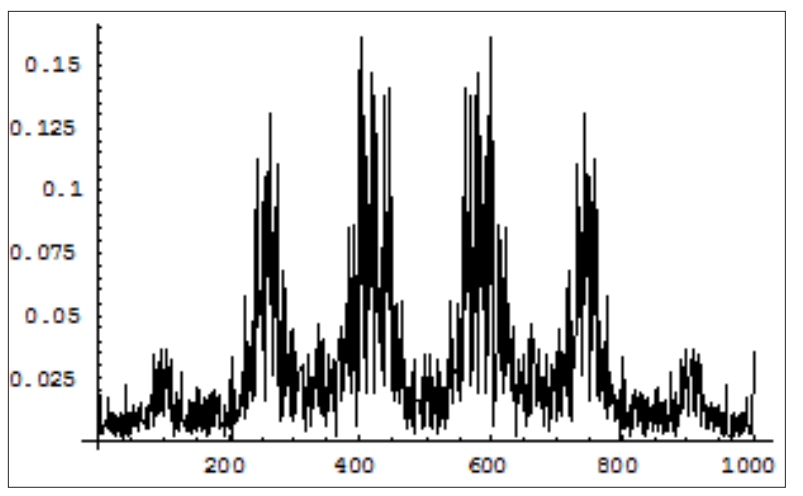

Figure 23

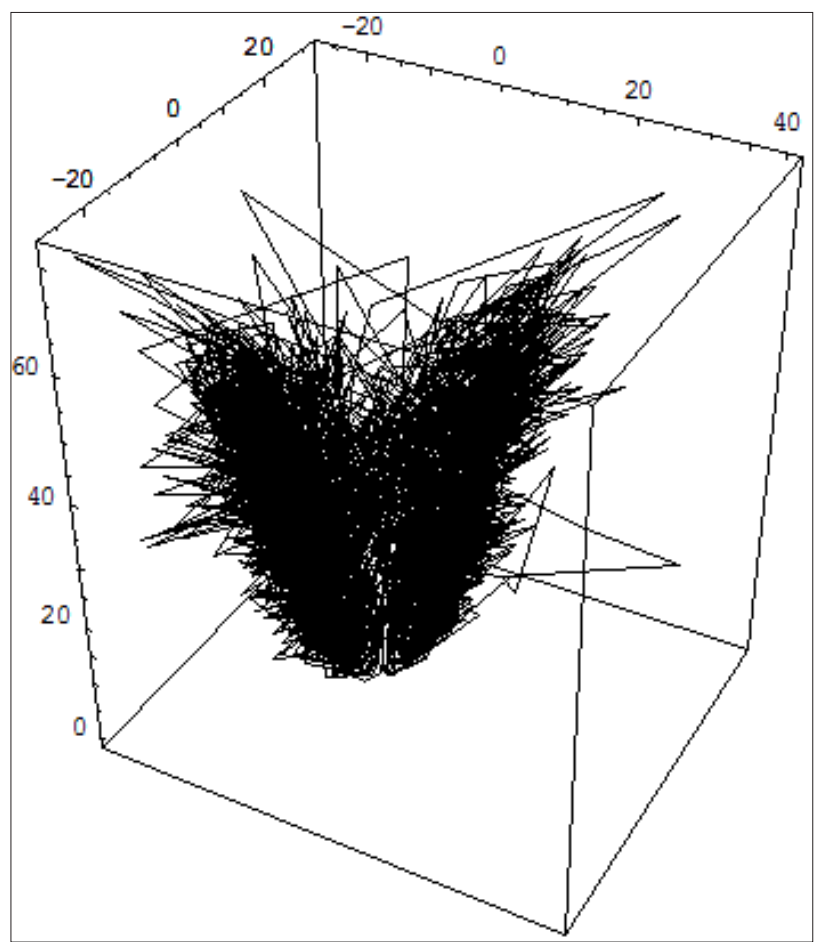

Figure 24: The 3D image of the functions $u, v, w$ in the system (23).



Figure 25: The functions in the system (23). 


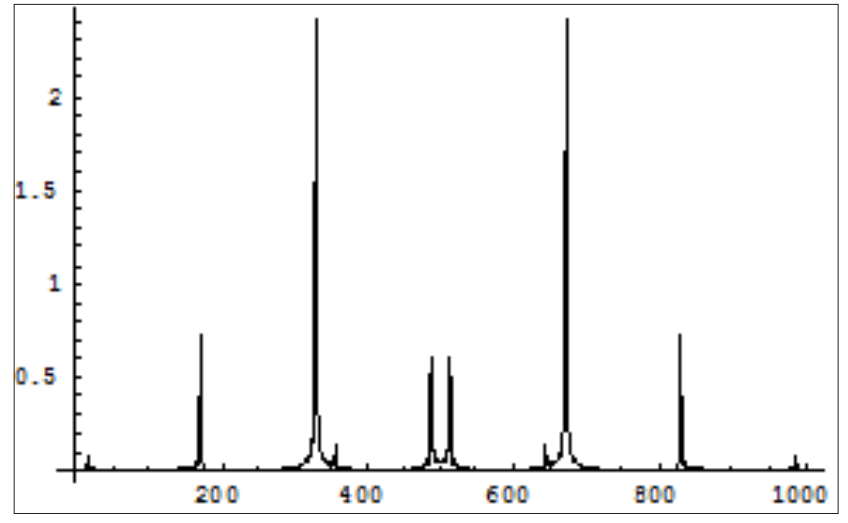

Figure 26

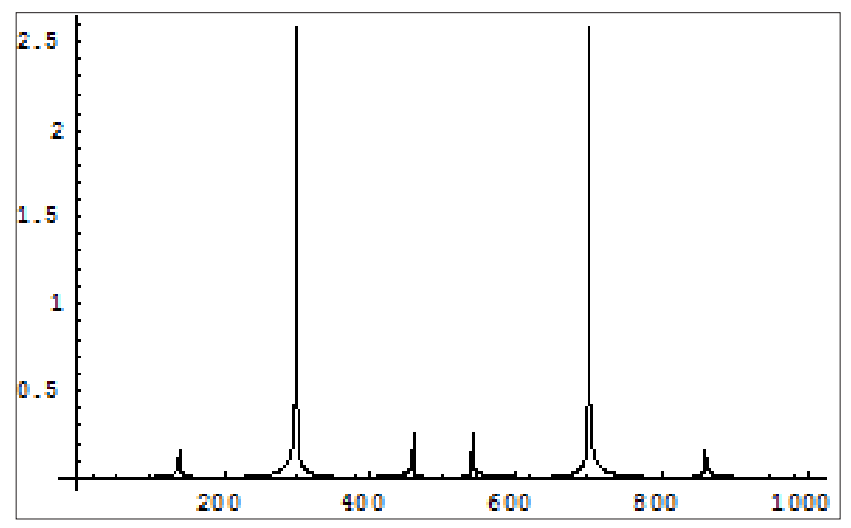

Figure 27

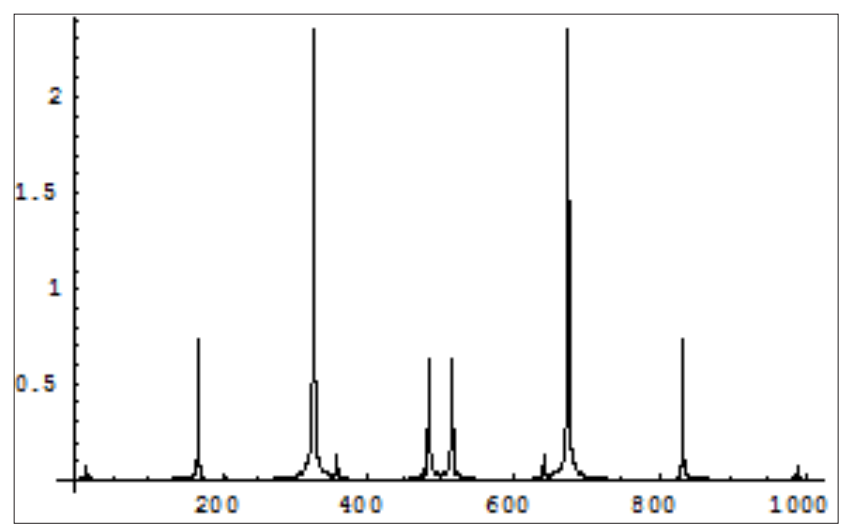

Figure 28

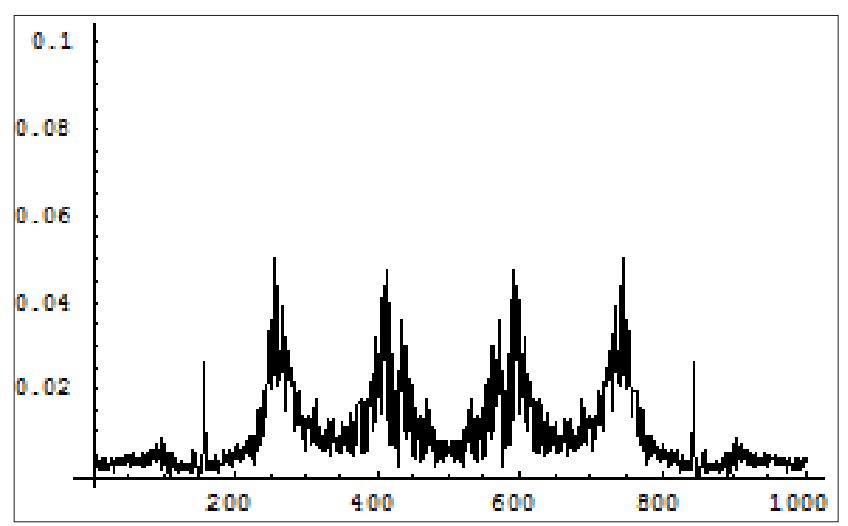

Figure 29

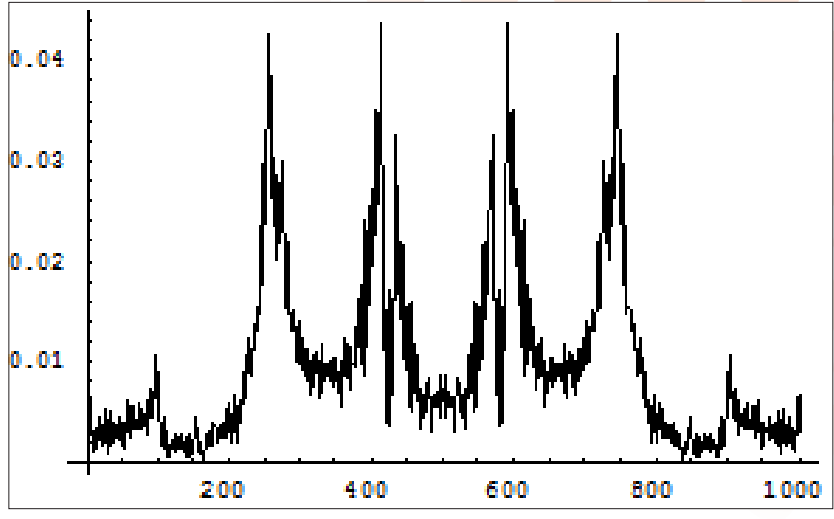

Figure 30

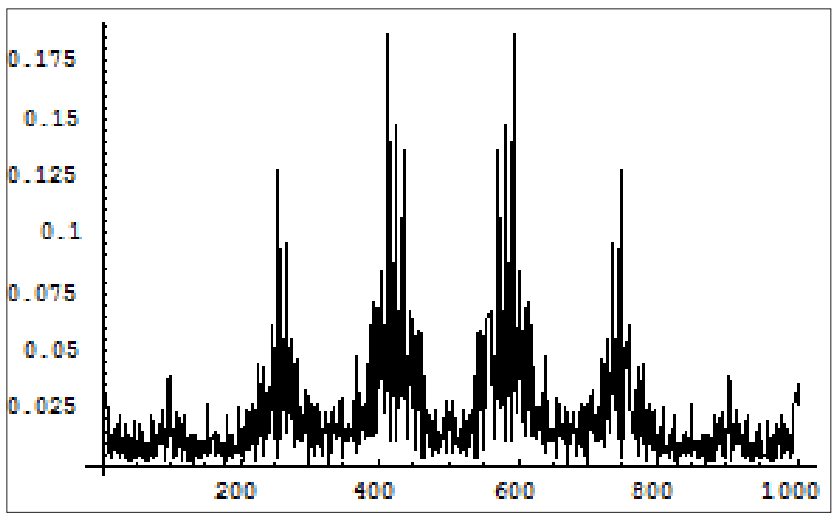

Figure 31

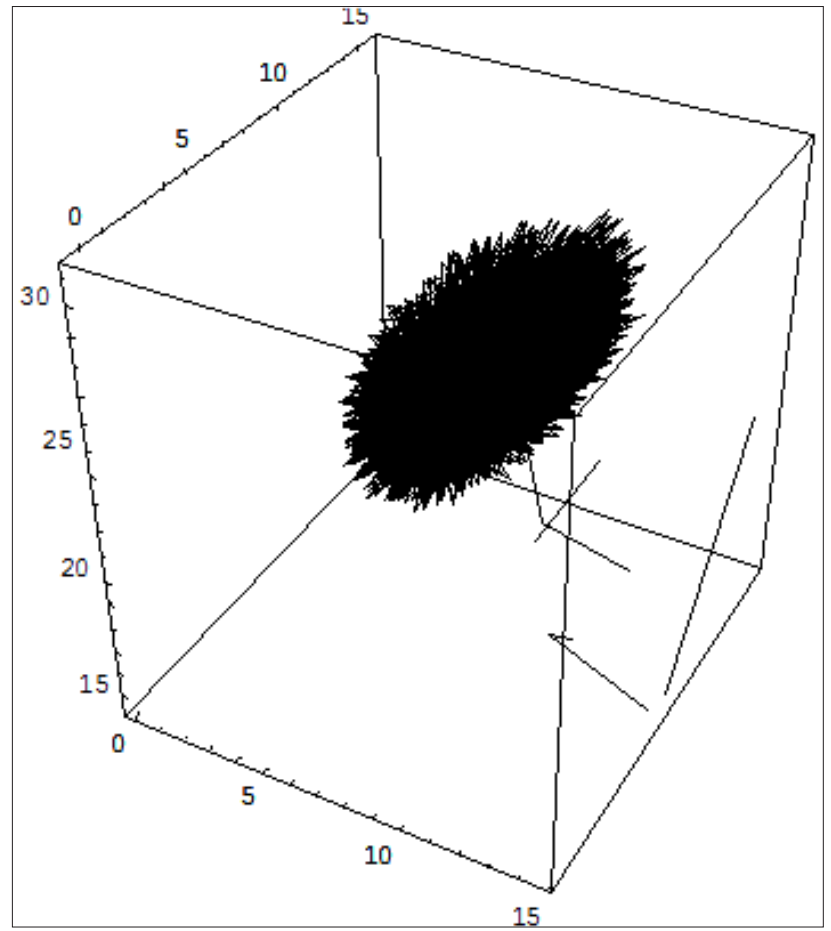

Figure 32: The $3 \mathrm{D}$ image of the functions in the system (23). 


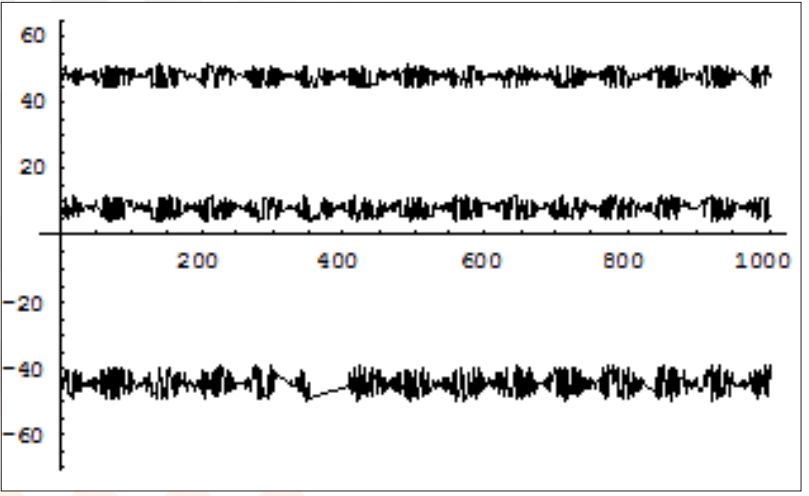

Figure 33: The functions in the system (23).

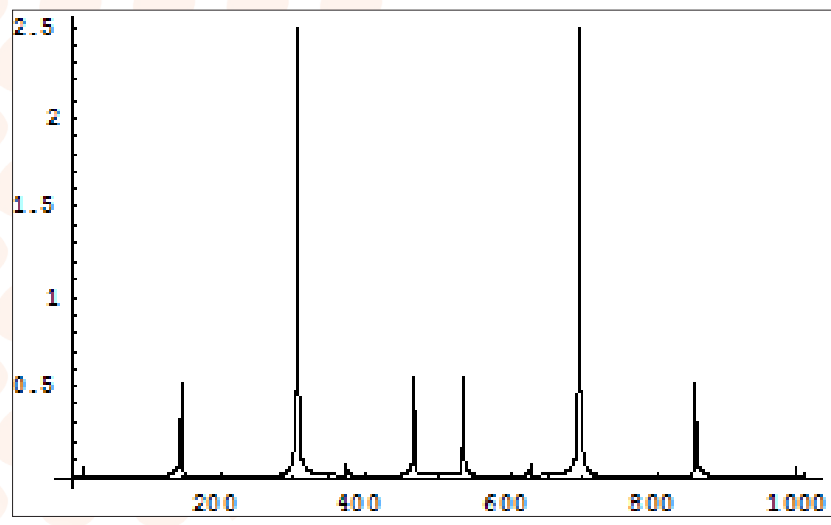

Figure 34

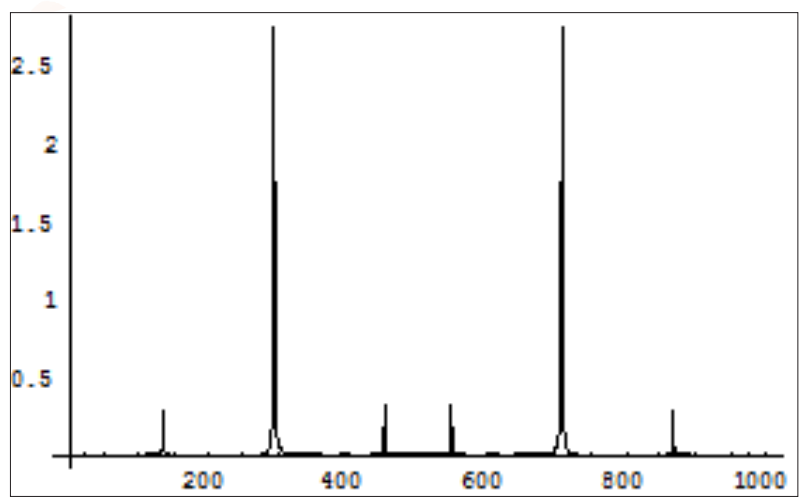

Figure 35

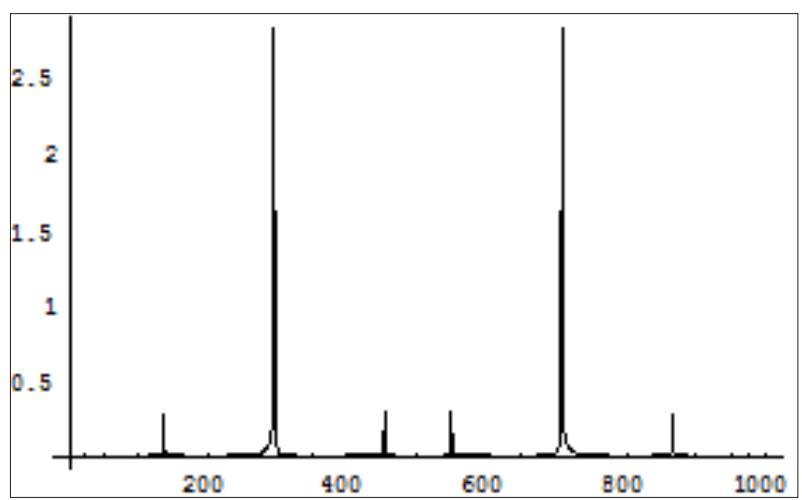

Figure 36

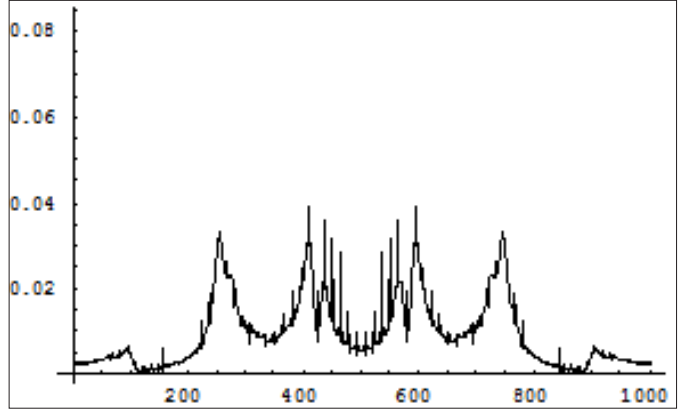

Figure 37

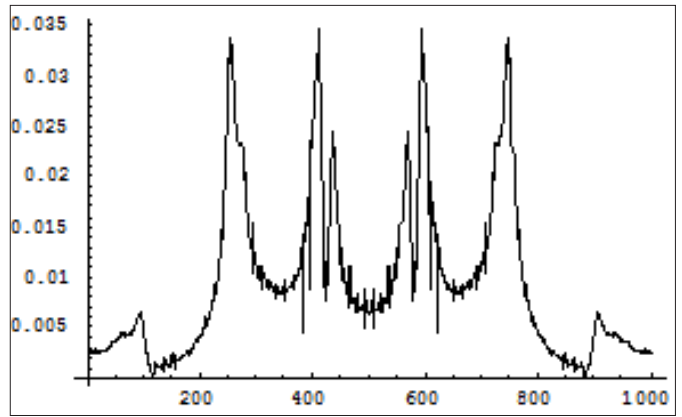

Figure 38

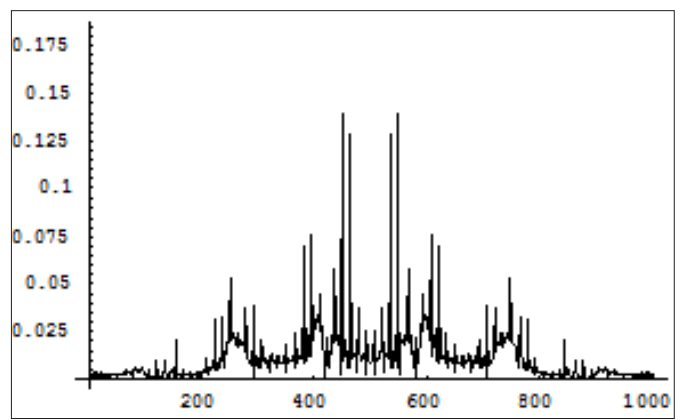

Figure 39

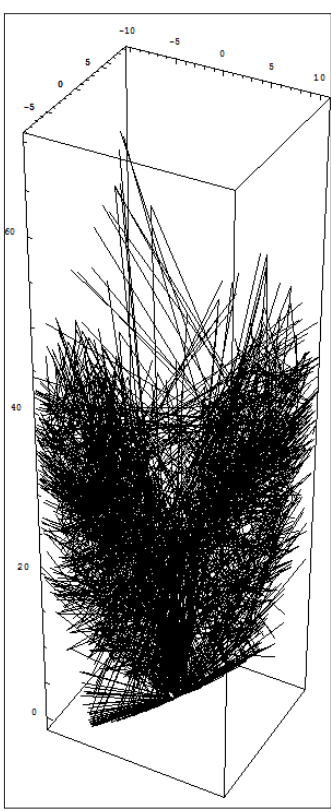

Figure 40: The $3 \mathrm{D}$ image of the functions in the system (23). 


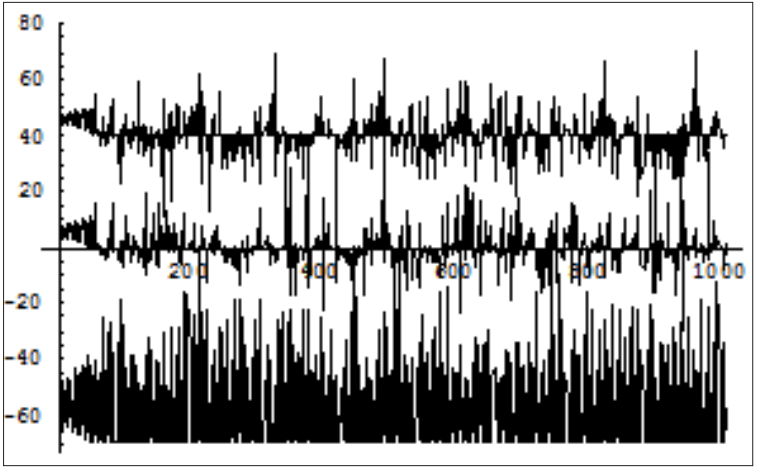

Figure 41: The functions in the system (23).

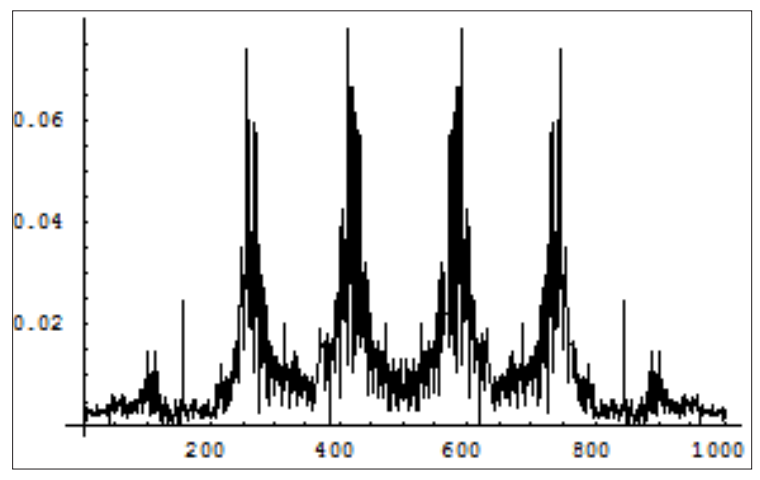

Figure 42



Figure 43

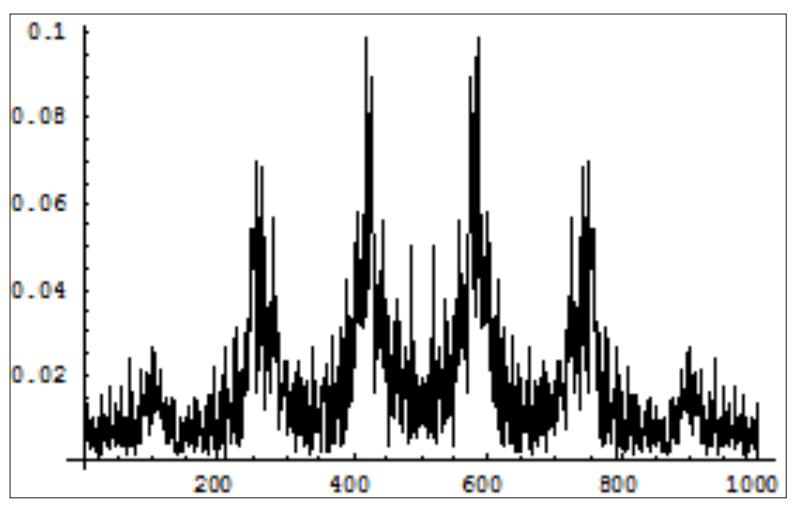

Figure 44

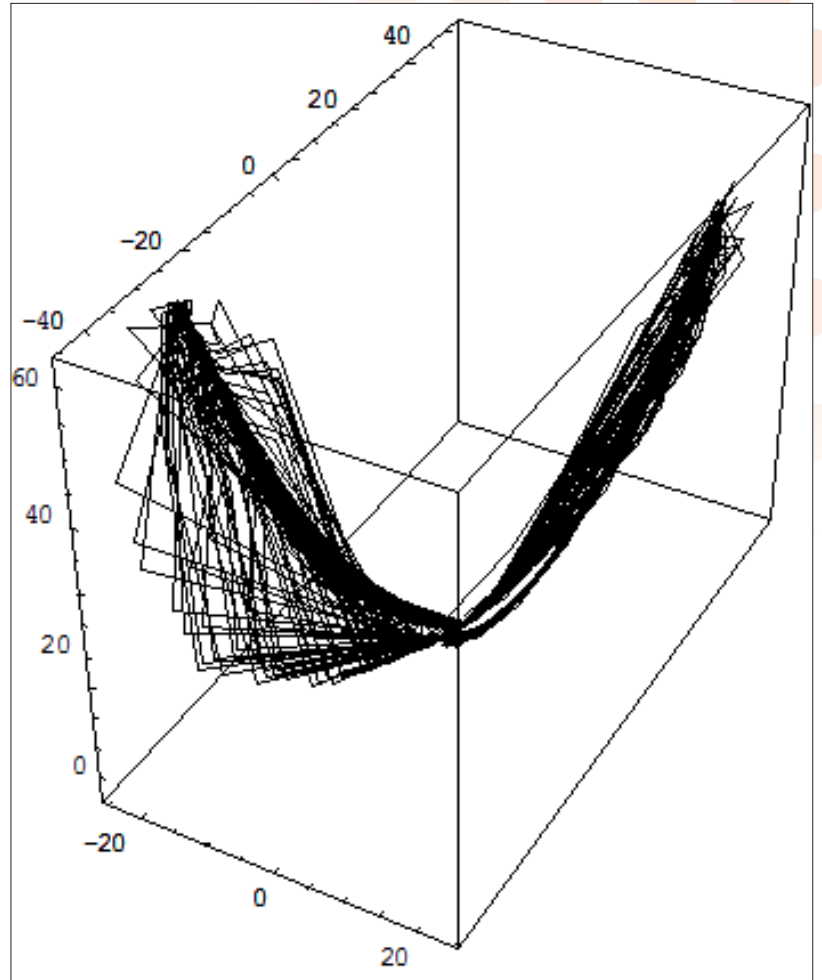

Figure 45: The 3 D image of the functions in the system (23).

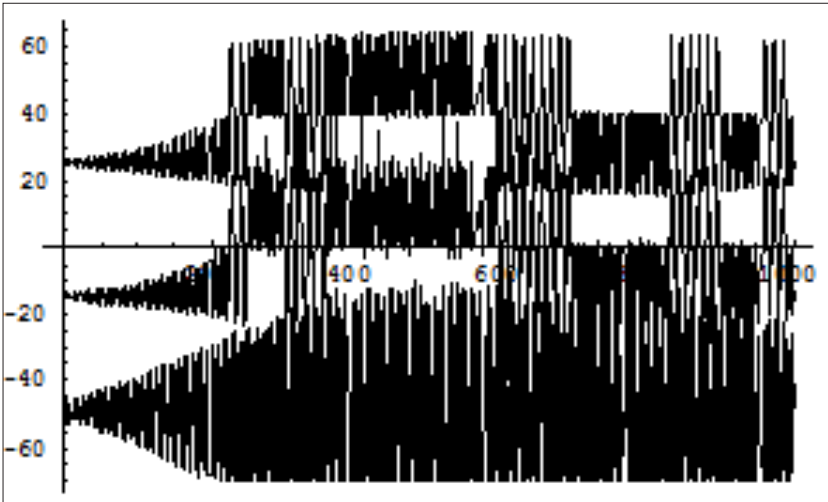

Figure 46: The functions $\mathrm{u}, \mathrm{v}, \mathrm{w}$ in the system (23).

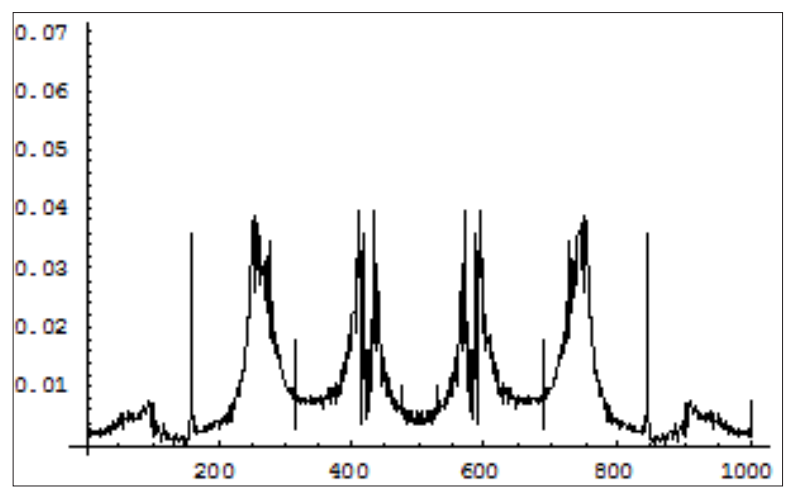

Figure 47 


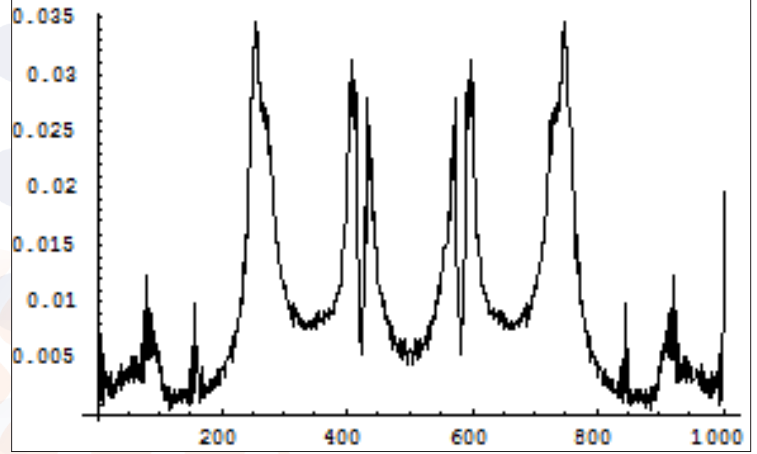

Figure 48

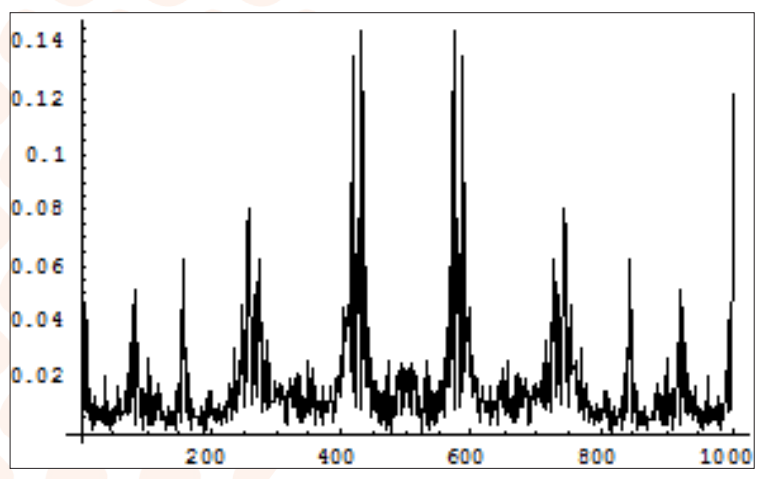

Figure 49

Analysis of the obtained solutions shows that the the main phonon generator forms the standing recurrence of the wave functions Fig.18, 19, 20, 26, 27, 28, 34, 35, 36 that don't differ from that of the triangular model: Fig.6, 7, 8, 9, 10, 11, 12, 13, 14 despite the different modes of the Lorenz model of physical vacuum: Fig.16, 17, 24, 25, 32, 33, 40, 41, 45, 46 which demonstrate a strange attractor (Fig.16.), an intermittency (Fig.24), a quasi regular attractor (Fig.32), a full stochastisity (Fig.40) and two interacting strange attractors (Fig.45). These modes proved to be statistically the most probable in the system. As far as the «governed» phonon generator standing recurrence pictures are concerned they revealed some dependence on the Lorenz model modes. In particular, the strange attractor mode (Fig.16) resulted in higher stability of the second generator standing recurrence structure (Fig.18, 19, 20). An intermittency mode (Fig.24) results in lowering of the resulting recurrence structure stability (Fig.26, 27, 28). A quasi regular attractor (Fig.32) brings the increasing of the intermediate spatial vibration amplitudes (Fig.37, 38, 39). The full stachastisity mode (Fig.40) results in the icreasing of the resulting recurrence structure stability (Fig.42, 43, 44). And the two attractor interaction mode (Fig.45) brings a nonuniformity into the structure of the resulting recurrences between three branches of the second generator (Fig.47, 48, 49). Worth mentioning that a considerable variation in the magnitudes of the Lorenz system (22) coefficients $\left(\sigma \rightarrow 1-10^{2}, \beta \rightarrow 1-10^{3}, b \rightarrow 1-10^{5}\right)$ didn't change qualitatively the structures of the obtained graphs.

The existing phenomenon of «blurring» of the recurrence phases takes place due to the incompleteness of the resonant interaction process between the first and second generator recurrences. Similar to the Fermi-Pasta-Ulam recurrences interaction features [9] the completeness arises after a number of the recurrence cycles. Moreover, an inevitable existance of gigantic phonon generators like stars and planets brings a distortion into resulting picture. For example carring out any gravity experiments on earth requires forming a partial standing recurrence of phonon functions in the earth structure after switching a described toroidal phonon generator. Such process ends when a traveling phonon wave turns into a standing one. That occurs after the time $t_{\text {travel }}=D_{\text {earth }} / V_{\text {sound }}=12714 \mathrm{~km} / 10 \mathrm{~km} /$ $\mathrm{sec} \approx 1271 \mathrm{sec} .$. The recurrence waves interact with a medium having random fluctuations in such a way that the dissipation is compensated through gaining energy from the random fluctuations of the medium [6].

\section{Conclusion}

The following conclusions can be made on the basis of the carried out theoretical research:

1. The developed mathematical model of the interaction process between two phonon generators through the medium of the physical vacuum (23) and its numerical study demonstrated that the gravity attraction between solid bodies (2) can be changed at the expense of the increasing or decreasing of the effective masses of phonons $(7,8)$, generated for example in a crystalline lattice of a ferromagnetic material.

2. A numerical study of the system (16) modeling the quantum dynamics of the wave functions in the phonon generator allowed to discover a new form of the quantum recurrence - a standing one. Differently from the classical Fermi-Pasta-Ulam recurrence the obtained one demonstrates only the phase and amplitude recurrences of the wave functions to their initial states. The standing recurrence seems not to have a certain spatial scale, which is imposed only by the dimensions of the phonon generators under consideration (even for example stars and planets).

3. According to the results of modeling a gravity attraction force represents a matter of mutual resonant interaction of the two body phonon standing recurrences existing in their structures. Since the interaction concerns only their mutual phase tuning, when a mass moves, the force acting on other masses is considered to adjust instantaneously to the new location of the displaced mass in accordance with the Isaac Newton's concept.

\section{References}

1. Encyclopaedia Britannica.

2. Callaway J (1958) Electron energy bands in solids: in Solid State Physics. Ed by F.Seitz and D. Turnbull. v 7, N.Y. 1958.

3. Pippard. A.B (1983) The Physics of Vibration. The Simple Vibrator in Quantum Mechanics. Cambridge University Press. 1983.

4. Teodorchik K.F (1952) Selfoscillating systems. Moscow, Leningrad, 1952. (in Russian). p 271. 
5. Ashcroft N.W., Mertin. N.D (1976) Solid State Physics p.519. Ed. By Holt, Rinehart and Winston N.Y. 1976.

6. Berezin. A. A (2003) Modeling of coherent and stochastic properties of physical and biological systems. (in Russian) Moscow, 2003.p 80.

7. Berezin.A.A. Quantum recurrence and its possible application. Website:fpu.nm.ru.

8. Fermi E., Pasta J., and Ulam S. In: Collected Papers of E. Fermi. 1955. vol. 2, p. 978.

9. Berezin.A.A (2004) Resonant interaction between the Fermi-Past-Ulam recurrences. Bulletin of Lebedev's Institute of Physics, 3: 41-50.

10. Saltzman. B. J. Atmos. Sci. 19, 329 (1962).

11. Lorenz. E.N. J. Atmos. Sci. 20, 130 (1963).

12. Lichtenberg. A. J. and Lieberman. M.A. Appl. Math.Sci. v 38. Springer-Verlag, 1991, p 450 - 469.

Copyright: $@ 2021$ A.A.Berezin. This is an open-access article distributed under the terms of the Creative Commons Attribution License, which permits unrestricted use, distribution, and reproduction in anymedium, provided the original author and source are credited. 